\title{
Einige Bemerkungen zur Zustandsänderung von relativistischen quantenmechanischen Systemen durch Messungen und zur Lokalitätsforderung
}

\author{
S. SCHLIEDER \\ Max-Planck-Institut für Physik und Astrophysik, München
}

Eingegangen am 27. November 1967

\begin{abstract}
A description of relativistic quantum mechanical systems is introduced, in which the states depend on the possible information of the observer. The possibility of information is limited by Einstein-causality for classical systems $\left(\mathscr{C}_{k l}\right)$. Observables belonging to measurements in finite regions of the Minkowskispace have a degenerate spectrum. The measurement of such an observable leads to a mixture; for the description of such a mixture we use a proposal first made by LÜDERS $(\mathscr{G})$. We assume the locality condition for the observables $(\mathscr{L})$, this means the commutativity of two observables measured in two relatively space-like regions. The main result is the equivalence of $(\mathscr{L}),(\mathscr{G}),\left(\mathscr{C}_{k l}\right)$ on the one side and $(\mathscr{G})$ and $(\mathscr{C})$ on the other side. $(\mathscr{C})$ is the Einstein-causality including quantum mechanical systems. The idealisation of a measurement as a point event is used for the derivation of this result.
\end{abstract}

\section{Einleitung}

Den Anstoß zu diesen Bemerkungen bildete die Frage, inwieweit die Lokalitätsforderung für relativistische Quantenfeldtheorien begründet erscheint, wenn sie mit den durch Meßeingriffe hervorgerufenen Störungen in Zusammenhang gebracht wird. Betrachtet man z. B. ein reelles skalares relativistisches Quantenfeld $A(x)$ und sieht es als ein Modell für ein observables Feld an, so wird meistens die Lokalitätsforderung

$$
[A(x), A(y)]_{-}=0,
$$

für $x-y$ raumartig verlangt [1]; $x$ bzw. $y$ sind Abkürzungen für die 4-Vektoren $x_{0}, x_{1}, x_{2}, x_{3}$ bzw. $y_{0}, y_{1}, y_{2}, y_{3}$.

Wenn Felder, welche an einem Streuprozeß beteiligt sind, die Lokalitätsforderung erfüllen, so kann man wichtige Analytizitätseigenschaften für die Streuamplituden (Dispersonsrelationen) herleiten. Diese Analytizitätseigenschaften sind im Einklang mit den experimentell gewonnenen Daten für Streuprozesse. Andererseits ist es natürlich nicht möglich, von den experimentellen Daten exakt auf analytische Eigenschaften der Streuamplituden zu schließen. Auch wenn dieses möglich wäre, ist nicht wohlbekannt, bis zu welchem Ausmaß Lokalitätseigenschaften der Felder 
aus vorgegebenen analytischen Eigenschaften der zugehörigen Streuamplituden folgen; daß man eine so strenge Forderung wie in (1) ableiten kann, ist sehr unwahrscheinlich.

Man mag daher unsicher sein, ob es zweckmäßig ist, die Klasse der relativistischen Quanten-Felder durch eine Forderung wie in (1) so stark einzuschränken, insbesondere, wenn man den Mangel an mathematisch definierten relativistischen Quantenfeldtheorien, welche echte Streuung aufweisen, vor Augen hat [2]. Es braucht so nicht überflüssig zu sein, den Zusammenhang zwischen der Lokalitätsforderung einerseits und Zustandsänderungen von relativistischen quantenmechanischen Systemen, welche durch Meßprozesse hervorgerufen werden, andererseits, ein Stück zu verfolgen.

Den hier erörterten Fragen kann man etwa am Beispiel eines relativistischen Observablen-Feldes, das in einem Hilbertraum wirkt, nachgehen. Hierzu betrachtet man z. B. beim Feld $A(x)$ die Linearkombinationen der Ausdrücke

$$
A\left(f_{1}\right) A\left(f_{2}\right) \ldots A\left(f_{j}\right) \Omega, \quad j=0,1,2, \ldots
$$

und vervollständigt diesen linearen Raum zu einem Hilbertraum (Gelfand-Neumark-Segal-Konstruktion). $\Omega$ ist der zyklische Vakuumzustand und $A\left(f_{k}\right)=\int A(x) f_{k}(x) d x$, wobei $f_{k}(x)$ einem geeigneten Raum von Testfunktionen angehört. Es ist aber auch möglich, ObservablenAlgebren zu betrachten [3], die von lokalen Observablenringen $\mathfrak{A}(R)$ erzeugt werden; $R$ bedeutet einen Teil des Minkowski-Raumes. An diese stellt man ebenfalls häufig die Forderung der Vertauschbarkeit der Operatoren, sofern sie zu raumartig zueinander gelegenen Gebieten gehören:

$$
[A, B]_{-}=0
$$

für $A \in \mathfrak{A}\left(R_{1}\right)$ und $B \in \mathfrak{A}\left(R_{2}\right)$ und $R_{1}$ raumartig zu $R_{2}$.

Eine Lokalitätsforderung, wie sie in (1) bzw. (2) erscheint, kürzen wir allgemein hier mit $(\mathscr{L})$ ab.

Sowohl bei der ersten wie bei der zweiten Art der Beschreibung können die Operatoren (bzw. die Elemente der Algebra) in zwei verschiedenen Interpretationen auftreten [4]. Betrachten wir z. B. $A(f)$ $==\int A(x) f(x) d x$. Wenn es möglich ist, das Wirken von $A(f)$ als eine physikalische Operation zu deuten, Operation z. B. am Zustand $\psi$ :

$$
\psi \rightarrow A(f) \psi,
$$

dann erscheint die Korrespondenz zwischen $\mathscr{T} r(f)(\mathscr{T} r$ gleich Träger $)$ und dem Gebiet, an dem die Operation stattfindet, weitgehend aufhebbar. Unter den üblichen Annahmen bilden bekanntlich $\left\{\mathscr{P}_{O} \ell\left(A\left(f_{j}\right)\right)\right\} \Omega$ mit den $\mathscr{T} r\left(f_{j}\right)$ in einem beliebigen offenen Teil $O$ des Minkowski-Raumes einen dichten Teilraum des Hilbertraumes [5]. Wenn andererseits $A(f)$ 
mit reellem $f$ einem selbstadjungierten Operator entspricht [6] und als Observable interpretiert wird, so hat man zur Motivierung der Lokalitätsbedingung vorauszusetzen, daß die Messung dieser Observablen in $\mathscr{T} r(f)$ stattfindet.

Wir wollen hier nicht die Frage in Angriff zu nehmen versuchen, ob man beide Interpretationen nebeneinander konsequent durchhalten kann. Den Überlegungen wird die zweite Interpretation zugrunde gelegt. Wir schließen uns dem für dieses Gebiet üblichen Brauch an und verwenden das Heisenbergbild in der Präzisierung, daß ein Element des Zustandsraumes als Zustand des Systems in seiner ganzen 4-dimensionalen Erstreckung interpretiert wird (,ZZustand sub specie aeternitatis" [7]), im weiteren abgekürzt als H.A.-Zustand. Ein Teil der Bemerkungen bezieht sich auf die Frage, in welcher Weise die Beschreibung durch H.A.Zustände erfolgen soll, wenn Messungen am System stattfinden.

Die Forderung der Lokalität an die Felder bzw. an die Observablenringe muß natürlich in Zusammenhang mit der Einstein-Kausalität gebracht werden, d. h. mit der Forderung, daß sich physikalische Effekte nicht mit größerer als mit Lichtgeschwindigkeit fortpflanzen können. Die Einstein-Kausalität wird zunächst als eine Forderung an die nichtquantenmechanischen Systeme der klassischen Physik aufgefaßt; sie sei hier mit $\left(\mathscr{C}_{k l}\right)$ abgekürzt.

Wir wollen, wo Genauigkeit erforderlich ist, zwischen experimentellem Eingriff und Beobachtung unterscheiden. $Z_{e}(a)$ soll den erfolgten oder auch geplanten Eingriff an der Stelle $a$ bedeuten, $Z(b)$ die Beobachtung des Systems an der Stelle $b$; d. h. ein bei $b$ befindlicher Beobachter setzt die bei $b$ erhältlichen Informationen in eine Zustandsbeschreibung um. Bei zwei raumartig zueinander erfolgenden Meßeingriffen, wie sie der Lokalitätsbedingung zugrunde liegen, sind wegen $\left(\mathscr{C}_{k l}\right)$ zwei verschiedene Experimentatoren $Z_{e}$, bzw. zwei verschiedene Apparate erforderlich. Für einen Beobachter $Z$ ist $\left(\mathscr{C}_{k l}\right)$ ebenfalls wesentlich, da durch diese Bedingung die Informationsmöglichkeit eingeschränkt und damit die $\mathrm{Zu}$ standsbeschreibung beeinflußt wird. An sich ist für die Zustandsbeschreibung auch bei komplizierten Meßprogrammen nur ein Beobachter $Z$ notwendig. Um die mögliche Abhängigkeit der Zustandsbeschreibung vom Ort und von der Geschwindigkeit des Beobachters darzulegen, überziehen wir jedoch den Minkowskiraum mit einem Feld von Beobachtern, die sich für einen festen Raumzeitpunkt noch durch verschiedene Geschwindigkeiten relativ zu einem gegebenen Koordinatensystem unterscheiden können.

Neben den Bedingungen $(\mathscr{L})$ und $\left(\mathscr{C}_{k l}\right)$ hat man eine Annahme über die Art der Gemenge zu machen, die nach Messung einer Observablen erzeugt werden, die zu einem selbstadjungierten Operator mit nichteinfachem Spektrum gehört. Dieser Fall liegt vor, wenn es sich um eine 
Observable handelt, deren Messung in einem endlichen Teil des Minkowski-Raumes erfolgt; nur solche Messungen sind ja realisierbar. Hier folgen wir einem Vorschlag, der zuerst von LüDers [8] gemacht wurde; wir kürzen ihn mit $(\mathscr{G})$ ab.

Die Einstein-Kausalität allgemein, d.h. unter Einbeziehung von quantenmechanisch behandelten Systemen werde mit $(\mathscr{C})$ bezeichnet. Das Hauptergebnis der folgenden Überlegungen besteht in Relationen, welche $(\mathscr{L}),\left(\mathscr{C}_{k l}\right),(\mathscr{G})$ und $(\mathscr{C})$ miteinander verknüpfen.

Die Resultate sind nicht ohne Idealisierungen der tatsächlich durchführbaren Meßeingriffe zu gewinnen. Es soll daher darauf verzichtet werden, diese Ergebnisse bereits hier abgetrennt von den übrigen Überlegungen vorwegzunehmen.

Wir wollen von dem einfachen Fall ausgehen, daß im Zustandsraum keine Überauswahlregeln (Superselection-Rules) vorhanden sind. Wir diskutieren hier nicht das physikalische Geschehen während eines Meßeingriffs [9], sondern nur den pauschalen Einfluß einer Messung. Das Problem der Erzeugung der in ein Meßprogramm einlaufenden Zustände ist ebenfalls ausgeklammert; nur am Ende findet sich hierzu eine kurze Bemerkung.

\section{Zustandsänderung durch Messungen und Heisenbergbild}

Zuerst soll hier an die verschiedenen wohlbekannten Interpretationsmöglichkeiten für den quantenmechanischen Formalismus im Hinblick auf die Wirkung von Meßprozessen im nichtrelativistischen Fall im Schrödingerbild erinnert werden. Dazu mag ein einfaches Beispiel dienen: Messung einer Observablen ${ }^{1} A$, die ein reines Punktspektrum mit einfachen Eigenwerten $\left\{a_{j}\right\}$ besitzt, zur Zeit $t=t^{*} ; \psi_{j}$ seien die Eigenzustände. Die einparametrige Schar $\varphi(t)=e^{-i H t} \varphi(0)$ (mit $H$ als Hamiltonoperator) stelle für $t<t^{*}$ die zeitliche Entwicklung des einlaufenden Systems dar, und es sei $\varphi\left(t^{*}\right)=\sum_{j} c_{j} \psi_{j}$.

Eine einparametrige Schar $\varphi(t)$ läßt zwei Interpretationen zu:

1. $\varphi(t)$ beschreibt die zeitliche Entwicklung eines einzigen Systems [10] $\Sigma$.

2. $\varphi(t)$ beschreibt die zeitliche Entwicklung einer Systemgesamtheit $G^{\Sigma}$ von $N$-Systemen, $N$ sehr groß, wobei sich jedes Einzelsystem in dem gleichen Zustand befindet.

Wir nehmen an, daß zur Zeit $t=t^{*}$ der Eigenwert $a_{m}$ im Falle der ersten Interpretation gemessen wird, und vergleichen die Aussagen von zwei Beobachtern $Z_{1}\left(t_{1}\right)$ mit $t_{1}<t^{*}$ bzw. $Z_{2}\left(t_{2}\right)$ mit $t_{2}>t^{*}$ miteinander. Die Beobachter sollen den Zustand des einlaufenden Systems kennen

1 Wir werden hier und im folgenden nicht immer, wie es korrekt wäre, zwischen Observablen und zugehörigen selbstadjungierten Operatoren unterscheiden. 
und wissen, daß zur Zeit $t=t^{*}$ die Messung von $A$ stattfinden soll bzw. stattgefunden hat.

$Z_{1}$ für $\Sigma: \Sigma$ wird zur Zeit $t^{*}$ mit der Wahrscheinlichkeit $w_{j}=\left|c_{j}\right|^{2}$ in $\psi_{i}$ übergehen.

$Z_{1}$ für $G^{\Sigma}: G^{\Sigma}$ wird zur Zeit $t^{*}$ in die Teilgesamtheiten $G_{1}^{\Sigma}, G_{2}^{\Sigma}, \ldots$, $G_{j}^{\Sigma}, \ldots$ mit den relativen Häufigkeiten $\frac{N_{1}}{N}, \frac{N_{2}}{N}, \ldots \frac{N_{j}}{N}, \ldots$ bzw. übergehen mit $w_{j}=\frac{N_{j}}{N}\left(G_{j}^{\Sigma}\right.$ für $t=t^{*}$ durch $\psi_{j}$ beschrieben $)$.

$Z_{2}$ für $\Sigma: \Sigma$ ging zur Zeit $t^{*}$ in $\psi_{m}$ über. Dabei ist angenommen, daß $Z_{2}$ das Meßergebnis zur Kenntnis nahm. Wenn das nicht der Fall war, dann macht $Z_{2}$ die Aussage: $\Sigma$ ging zur Zeit $t^{*}$ mit der Wahrscheinlichkeit $w_{j}=\left|c_{j}\right|^{2}$ in $\psi_{j}$ über.

$Z_{2}$ für $G^{\Sigma}: G^{\Sigma}$ wurde zur Zeit $t^{*}$ in die Teilgesamtheiten $G_{1}^{\Sigma}, G_{2}^{\Sigma}, \ldots$, $G_{j}^{\Sigma}, \ldots$ mit den relativen Häufigkeiten $w_{j}$ zerlegt. Oder formal entsprechend der Nichtablesung des Meßergebnisses für $\Sigma$ : Die Teilgesamtheiten $G_{1}^{\Sigma}, G_{2}^{\Sigma}, \ldots, G_{j}^{\Sigma}, \ldots$ werden erneut zusammengefaßt und durch ein Gemisch mit dem statistischen Operator $W=\sum_{k} w_{k} P_{k}$ beschricben; $P_{k}$ ist der Projektor auf den Unterraum zu $a_{k}$ (hier eindimensional).

Die weiter unten auftretenden Sachverhalte kann man sich mit Vorteil in beiden Interpretationen klar machen. Der Annahme des Auftretens eines Eigenwertes $a_{m}$ für das Einzelsystem $\Sigma$ entspricht die Auswahl der Teilgesamtheit $G_{m}^{\Sigma}$ für $G^{\Sigma}$. Die zunächst etwas künstlich erscheinende Annahme, daß die Messung zwar stattgefunden hat, das Meßergebnis jedoch nicht zur Kenntnis genommen wurde, spielt später, wenn die durch $\left(\mathscr{C}_{k l}\right)$ bedingte Beschränkung der Informationsmöglichkeit betrachtet wird, eine wichtige Rolle.

Die im Schrödingerbild vorhandenen Begriffsbildungen reichen also im nichtrelativistischen Fall aus, Zustandsänderungen als Folge von Meßeingriffen zu beschreiben. Die Geschichte des Systems setzt sich aus Abschnitten zusammen. In jedem Zeitabschnitt $t_{j}<t<t_{j+1}$ ist das System ungestört und wird etwa durch eine Schar

$$
e^{-i I I\left(t-t_{j}\right)} \varphi_{j}\left(t_{j}\right), \quad t_{j}<t<t_{j+1}
$$

beschrieben. Auf den Grenzen der Abschnitte finden Messungen statt. Um an dieser Stelle unnötige Schwierigkeiten zu vermeiden, haben wir dabei angenommen, daß Observable mit einfachem Spektrum gemessen werden, und die Reduktion der Gemische durch Kenntnisnahme des Meßergebnisses am System $\Sigma$ oder durch Auswahl einer Teilgesamtheit gemäß dem Meßergebnis, sofern man von einer Systemgesamtheit $G^{\Sigma}$ ausgeht, erfolgt.

Bei Verwendung von H.A.-Zuständen scheint es dagegen zunächst an den notwendigen Begriffen zu fehlen, eine durch einen Meßeingriff 
hervorgerufene Zustandsänderung zu beschreiben. Meßeingriffe gehen in gewissen endlichen Raumbereichen und Zeitintervallen (abstrahiert zu gewissen Zeitpunkten) vor sich. Der Formalismus bietet jedoch nur die Ersetzung eines H.A.-Zustandes durch einen anderen an, sofern man eine Operation betrachtet:

$A \psi_{H A}=\varphi_{H A}$. Ebenso kann bei der Beschreibung eines Meßeingriffs ein H.A.-Zustand durch ein Gemisch oder - wenn man dieses reduziert durch eine Komponente des Gemisches ersetzt werden. Dabei läßt sich der Zeitpunkt des Übergangs von einem Zustand zu einem anderen an Hand der H.A.-Zustände allein nicht vermerken.

Wir wollen pointiert zusammenfassen:

(i) Ein System $\Sigma$ (oder auch Systemgesamtheit $G^{\Sigma}$ ), an dem gemessen wird, soll durch H.A.-Zustände $\left\{\psi_{j}\right\}$ beschrieben werden ${ }^{2}$. Jedes $\psi_{j}$ bezieht sich auf das ganze Zeitintervall $-\infty<t<+\infty$ und bedeutet in dieser Zeit den tatsächlichen Zustand des Systems.

(ii) Die Meßeingriffe gehen in endlichen Teilen des MinkowskiRaumes vor sich.

(iii) Ein Meßeingriff ändert im allgemeinen den Zustand des Systems.

Offensichtlich sind (i), (ii) und (iii) im Widerspruch zueinander.

Auf (ii) kann man überhaupt nicht, auf (iii) nicht bei einer Quantentheorie in der üblichen Formulierung verzichten. Deshalb wird (i) etwas modifiziert.

Ein H.A.-Zustand $\psi$ soll im allgemeinen nicht in seiner ganzen 4-dimensionalen Erstreckung den Zustand eines physikalischen Systems darstellen, an dem Messungen stattfinden. Wir bezeichnen einen Teil des Minkowski-Raumes, in dem die Beschreibung $\psi$ relevant für den Zustand des Systems ist, als ein $M$-Gebiet. Es seien $\left\{M_{j}\right\}$ irgendwelche $M$-Gebiete. Wir wollen diese bei den Zuständen vermerken. $\psi\left(M_{j}\right)$ bedeutet: Im Gebiet $M_{j}$ hat sich ein System so verhalten, wie ein anderes System, an dem keine Messungen erfolgten, im Zustand $\psi$. Keine Messungen ist so zu verstehen: Die Präparierung im Zustand $\psi$ wurde zu einer für alle Fragestellungen genügend frühen Zeit vorgenommen. $\psi$ ist gleichbedeutend mit $\psi(E)$, wenn $E$ den Minkowski-Raum bedeutet. Die Geschichte des Systems, an dem Messungen stattfinden, soll dann so beschrieben werden ${ }^{3}$ :

$$
\left\{\psi_{j}\left(M_{j}\right)\right\} \text { mit } \bigcup_{j} M_{j}=E, \quad M_{j} \cap M_{k}=\emptyset \text { für } j \neq k .
$$

An die Stelle der $\psi_{j}$ können auch Gemische mit statistischen Operatoren $W_{j}$ treten.

2 Index H.A. fortan weggelassen.

${ }^{3} \cup$ steht für Mengenvereinigung, $\cap$ für Durchschnittsbildung, $\theta$ für die leere Menge. 
Eine Überdeckung von $E$ durch Mengen $\left\{M_{j}\right\}$ in der beschriebenen Bedeutung heiße eine $M$-Überdeckung. Eine Mannigfaltigkeit $\left\{\psi_{j}\left(M_{j}\right)\right\}$, welche die Geschichte des Systems beschreibt, werde eine $M$-Karte genannt.

Die Meßeingriffe sind wieder so idealisiert, als ob sie zu einem Zeitpunkt stattfinden. Im nächsten Abschnitt sollen die Grenzen zwischen den $M$-Gebieten im relativistischen Falle definiert werden.

\section{Einflußgebiet einer Messung}

Betrachten wir zuerst ein nicht-statistisch behandeltes klassisches System, z. B. das elektromagnetische Feld im sonst materiefreien Raum. Das Feld, an dem keine Messungen stattfinden mögen, wird beschrieben durch eine bestimmte homogene Lösung (modulo einer Eichtransformation) der Gleichung

$$
\left.A_{v}(x)=0 \quad \text { (Nebenbedingung } \partial^{\prime \prime} A_{v}=0\right)
$$

Die Lösung kann durch die Anfangswerte auf einer Hyperfläche z. B. $x_{0}=\hat{x}_{0}$

$$
\varphi_{v}(\mathrm{x})=A_{v}\left(\hat{x}_{0}, \mathrm{x}\right), \quad \chi_{v^{\prime}}(\mathrm{x})=\dot{A}_{v}\left(\hat{x}_{0}, \mathrm{x}\right),
$$

charakterisiert werden.

Findet dagegen am Felde eine Messung statt, etwa durch Einbringung einer Probeladung an der Stelle $x=x^{*}$, so wird die Homogenität des Lösungscharakters gestört. In der Idealisierung des Meßeingriffs zu einem punktförmigen Meßeingriff bei $x^{*}$ sind zu $A_{v}\left(x_{0}^{*}, \mathbf{x}\right), \dot{A}_{v}\left(x_{0}^{*}, \mathbf{x}\right)$, wie sie sich nach der homogenen Gleichung ergeben, noch Distributionen, welche in $x^{*}$ konzentriert sind, zu addieren. Diese Distributionen hängen von der Art der Messung ab und brauchen hier nicht spezifiziert zu werden. Für das Gebiet $x_{0}>x_{0}^{*}$ ist dann die homogene Lösung relevant, die zu den Anfangswerten

$$
A_{v}\left(x_{0}^{*}, \mathrm{x}\right)+T_{v}\left(x^{*}\right), \quad \dot{A}_{v}\left(x_{0}^{*}, \mathbf{x}\right)+U_{v}\left(x^{*}\right)
$$

gehört; $T_{v}$ und $U_{v}$ sind die mit dem Meßeingriff verbundenen Distributionen.

Als wichtiger Zug der gestörten Lösung erscheint hier, daß die Störung auf den Vorwärtslichtkegel zum Punkte $x^{*}$ beschränkt ist. In dem speziell betrachteten Falle beschränkt sich die Störung auf den Mantel des Lichtkegels. Bei Problemen, bei denen sich die Störungen auch mit kleineren Geschwindigkeiten als mit Lichtgeschwindigkeit ausbreiten können, wird im allgemeinen auch das Innere des Lichtkegels erfaßt.

Die Verhältnisse werden anders, wenn man klassische Systeme in Betracht zieht, über die nur statistische Aussagen vorliegen. Es ist nicht schwer, sich Beispiele auszudenken, bei denen durch eine Messung an 
einer Stelle $x^{*}$ infolge der hierdurch gewonnenen Information die Beschreibung des Systems im Halbraum $x_{0}>x_{0}^{*}$ eine Änderung erfährt gegenüber der Beschreibung des Systems, wenn die Messung nicht stattgefunden hätte.

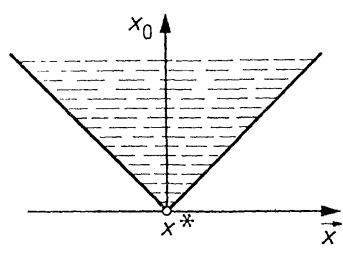

Fig. 1. Einflußgebiet einer Messung im Punkte $x^{*}$ bei einem nicht-statistischen klassischen System
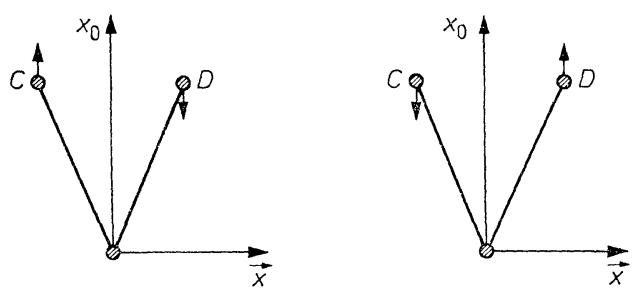

Fig. 2. Das von Aharovov und BoHn gegebene Beispiel

Bei nichtrelativistischen quantenmechanischen Systemen, deren Behandlung ja statistische Züge aufweist, wird man ebenfalls im allgemeinen den Halbraum $x_{0}>x_{0}^{*}$ als Einflußgebiet einer Messung bei $x^{*}$ erwarten. Hier kann jedoch bei gewissen Beispielen die Änderung des Zustandes infolge von Messungen so unerwartet ausfallen, daß einige Autoren von einem Paradoxon sprechen. Das sog. Einstein-PodolskyRosen-Paradoxon [11] ist bis in neuere Zeit in der Literatur immer wieder diskutiert worden [12]. Wir wollen hier an ein von AHaronov und Borrm [13] gegebenes Beispiel erinnern: Ein Molekül mit Spin 0 sei in Ruhe und zerfalle in zwei Teilchen $C$ und $D$, welche der Einfachheit halber verschieden angenommen werden, von denen jedes Spin 1/2 besitzt. Man setzt für das Molekül an:

$$
\psi=\text { const. }\left(\psi_{+}(C) \psi_{-}(D)-\psi_{-}(C) \psi_{+}(D)\right),
$$

$\psi_{+}$bzw. $\psi_{-}$haben die Komponenten in positiver bzw. negativer Spinrichtung. Nach dem Zerfall wartet man, bis die Teilchen praktisch dynamisch getrennt sind.

I. Man mißt zur Zeit $x_{0}=x_{0}^{*}$ die Spinkomponente von $C$ in $z$-Richtung. Man findet etwa $J_{z}^{C}=-\frac{1}{2}$. In diesem Augenblick hat man Gewiß- 
heit, daß $J_{z}^{D}=+\frac{1}{2}$ sein muß. Der veränderte Zustand ist durch $\psi_{--}(C) \psi_{+}(D) \mathrm{zu}$ beschreiben. Das Einflußgebiet der Messung am Orte von $C$ umfaßt auch den raumartig hierzu gelegenen Ort von $D$.

II. Hätte man zur Zeit $x_{0}=x_{0}^{*}$ die Spinkomponente von $C$ in $x$-Richtung gemessen und z. B. $J_{x}^{C}=+\frac{1}{2}$ gefunden, so wäre $D$ in den Eigenzustand $\psi_{-}^{\prime}-(D)$ zu $J_{x}^{D}=-\frac{1}{2}$ übergeführt worden.

Es ist zu vermerken, daß die Wahl der Richtung des Magnetfeldes, mit dem man die Spinrichtung von $C$ mißt (bei I $z$-Richtung, bei II $x$-Richtung) ebenfalls erst nach dynamischer Trennung von $C$ und $D$ erfolgen kann.

Systeme analoger Struktur sind der Zerfall von

$$
\begin{array}{r}
\text { Positronium } \rightarrow 2 \text { Photonen [14] } \\
\pi^{0} \text {-Meson } \rightarrow 2 \text { Photonen [15] } \\
\text { das } 2 \text {-Mesonsystem } K_{0}, \bar{K}_{0}[16] .
\end{array}
$$

An die Stelle der korrelierten Spinrichtungen treten in den beiden ersten Fällen korrelierte Polarisationszustände der Photonen, im zweiten Fall Korrelationen zwischen $K_{0}$ und $\bar{K}_{0}$ oder zwischen der kurz- und langlebigen Komponente des 2-Mesonsystems. Die Beispiele machen deutlich, daß die Veränderungen des Zustandes im Einflußgebiet der Messung auch besonders in den Teilen des Einflußgebietes, welche raumartig zum Orte des Meßeingriffs liegen, nicht nur als eine Beschreibung der verbesserten Kenntnis von dem System interpretiert werden können; siehe hierzu die beiden Meßanordnungen von I. und II. von oben mit sich gegenseitig ausschließenden Aussagen über die Eigenzustände von D. Eine Zustandsbeschreibung kann eine Reihe von Korrelationen zwischen den einzelnen Teilen des Systems beinhalten (im gewählten Beispiele Korrelationen zwischen den $z$-Komponenten bzw. $x$-Komponenten der Spins), wobei sich die Verifizierungen durch Messungen für zwei verschiedene Korrelationen ausschließen können. Das Herausgreifen einer der Korrelationen durch entsprechende Messung an einem Teil des Systems hat dann als Konsequenz im allgemeinen ein anscheinend der EinsteinKausalität widersprechendes Verhalten des anderen Teils des Systems zur Folge. Dieser Zug ist bei klassischen statistischen Systemen nicht vorhanden, bei denen raumartig zum Meßangriff gelegene Zustandsänderungen als Verbesserung der Kenntnis von dem bereits vor der Messung vorhandenen Zustand des Systems erscheinen.

Nunmehr soll in die Betrachtung das Wirken der Lorentzgruppe einbezogen werden. Wir nehmen also an, daß die inhomogene Lorentzgruppe im Hilbertraum unitär dargestellt wird. (An dieser Stelle würde auch die homogene Lorentzgruppe als Symmetriegruppe genügen.) Nun- 
mehr kann jede raumartige Hyperebene als Träger für Korrelationen der oben beschriebenen Art auftreten.

Nehmen wir z. B. den einfachen Fall, daß bei $x^{*}$ eine Messung stattfindet und sich verschiedene Beobachter bei $x *$ befinden. Ein im Koordinatensystem $K_{x}$ ruhender Beobachter wäre geneigt, eine $M$-Karte

$$
\left\{\psi_{1}\left(M_{1}\right), \psi_{2}\left(M_{2}\right)\right\} \text { mit } \begin{aligned}
M_{1} & =\left\{x ; x_{0}<0\right\} \\
M_{2} & =\left\{x ; x_{0}>0\right\}
\end{aligned}
$$

$\left(x^{*}=0\right.$ gesetzt) zu verwenden; dabei beschreibt $\psi_{1}$ den einlaufenden, $\psi_{2}$ den durch die Messung erzeugten Zustand. Ein im Koordinatensystem $K_{x^{\prime}} x_{v}^{\prime}=\Lambda_{v}^{\mu} x_{\mu}$ ruhender Beobachter würde dagegen die Beschreibung

$$
\left\{\begin{aligned}
\left\{\psi_{1}^{\prime}\left(M_{1}^{\prime}\right), \psi_{2}^{\prime}\left(M_{2}^{\prime}\right)\right\} \text { mit } \quad M_{1}^{\prime}=\left\{x^{\prime} ; x_{0}^{\prime}<0\right\} \\
M_{2}^{\prime}=\left\{x^{\prime} ; x_{0}^{\prime}>0\right\}
\end{aligned}\right.
$$

wählen. Übrigens wollen wir im folgenden die hier nicht interessierende Transformation der Zustände $\psi_{j} \rightarrow \psi_{j}^{\prime}$ unterlassen, d. h. alle Beobachter wählen zur Beschreibung der Zustände das gleiche Koordinatensystem und nicht ihr Ruhsystem; hier interessieren nur die Grenzflächen zwischen den $M$-Gebieten.

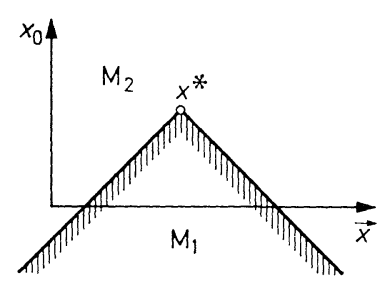

Fig. 3. Invariante $M$-Überdeckung für eine Messung bei $x^{*}$

Eine von der Geschwindigkeit der Beobachter abhängige $M$-Bedeckung wie oben in dem einfachen Beispiel beschrieben, führt zu einer unnötig komplizierten Darstellung. Wie sich weiter herausstellen wird, kann man eine invariante $M$-Bedeckung wählen. Es wird daher in dem Beispiel $M_{1}=R_{x^{*}}$, mit $R_{x^{*}}$ Rückwärtskegel zu $x^{*}, M_{2}=\left(R_{x^{*}}\right)_{c}$, wobei $\left(M_{j}\right)_{c}$ die Komplementärmenge zu $M_{j}$ bedeutet. Die Meßeingriffe sind zu punktförmigen Eingriffen idealisiert. In Wirklichkeit sind infolge der Dauer der Messung und der Ausdehnung des Meßapparates die Grenzen zwischen einzelnen $M_{j}$ verschwommen. Eine Unterscheidung, ob z. B. $R_{x^{*}}$ als abgeschlossen oder offen angesehen werden soll, hat daher physikalisch wenig Sinn. Um eine einheitliche Darstellung zu erreichen und die Bedingung $M_{j} \cap M_{k}=\emptyset, j \neq k$ zu erfüllen, nehmen wir als Konvention: $R_{x^{*}}$ ist abgeschlossen, $\left(R_{x^{*}}\right)_{c}$ infolgedessen offen. 
Ehe kompliziertere Meßprogramme diskutiert werden, sollen im nächsten Abschnitt die Messung von Observablen mit nicht-einfachem Spektrum und die resultierenden Gemischbildungen diskutiert werden.

\section{Zur Gemischbildung durch Messung von Observablen mit nicht-einfachem Spektrum}

Betrachten wir z. B. das skalare neutrale Feld $A(x)$, und bilden wir $A(f)=\int A(x) f(x) d x$ mit $f$ reell und $\mathscr{T} r(f)$ kompakt.

Wir wollen annehmen, daß dieser hermitische Operator zu einem selbstadjungierten Operator, welcher die gleichen Lokalitätseigenschaften besitzt wie der ursprüngliche Operator, fortgesetzt werden kann. Falls die Borchers-Zimmermann-Bedingung [6] gilt, ist dieses jedenfalls der Fall. Es ist wohlbekannt und auch leicht zu beweisen, daß dann bei Annahme der Lokalitätsbedingung der Operator $A(f)$ (wir bezeichnen den fortgesetzten Operator etwas nachlässig genauso wie den ursprünglichen) kein einfaches Spektrum besitzen sollte; andernfalls liegt ein $c$-Zahl-Feld vor. Die gleiche Bemerkung gilt für die lokalen selbstadjungierten Operatoren in einer Formulierung mit Hilfe lokaler Observablen-Ringe.

Wir haben es bei unseren Überlegungen mit lokalen Messungen zu tun und wollen hier daran festhalten, daß die zugehörigen Observablen aus den Feldern mit Hilfe von Testfunktionen gewonnen werden, deren Träger ebenfalls im Gebiet der Messung lokalisiert sind. Wir müssen also davon ausgehen, daß diese Observablen durch selbstadjungierte Operatoren mit nicht-einfachem Spektrum dargestellt werden.

Betrachten wir zunächst den Fall eines selbstadjungierten Operators $A$ mit reinem Punktspektrum. Es seien $\left\{a_{k}\right\}$ seine Eigenwerte $\left(a_{k} \neq a_{l}\right.$ für $k \neq l), P_{k}$ die Projektionsoperatoren auf die zugehörigen Unterräume, welche nun im allgemeinen nicht mehr eindimensional sind.

Es gibt für die Änderung des Zustands infolge der Messung einer solchen Observablen $A$ zwei verschiedene Ansätze: Einen bei den ursprünglichen Diskussionen über den quantenmechanischen Meßprozeß von J. v. NEUMANN [17] vorgeschlagenen und einen zweiten, der später von G. LÜDERs [8] gemacht wurde. Um die beiden Vorschläge gegenüberzustellen, nehmen wir der Einfachheit halber an, daß vor der Messung der reine Zustand $\varphi$ vorhanden ist, und daß das Meßergebnis abgelesen und damit zur Reduktion des Zustandes herangezogen wird. Der abgelesene Eigenwert sei etwa $a_{l}$, und es sei $P_{l} \boldsymbol{H}$ der zugehörige $n$-dimensionale Unterraum.

Im Vorschlag von J. v. Neumann wird angenommen, daß durch den Meßapparat, mit dem $A$ gemessen wird, ein bestimmtes Orthonormalsystem von Eigenvektoren $\varphi_{k m}$ ausgezeichnet ist, mit

$$
A \varphi_{k m}=a_{k} \varphi_{k m} \text {. }
$$


Es sei

$$
\varphi=\sum_{l, m} c_{l m} \varphi_{l m} \quad \text { mit } \sum_{l, m}\left|c_{l m}\right|^{2}=1 .
$$

Die Auszeichnung des Orthonormalsystems macht sich bei der Änderung des Zustands infolge der Messung bemerkbar. Bei den hier gemachien Annahmen entsteht aus $\varphi$ das Gemisch (es werden hier für Zustände bzw. Gemische die zugehörigen statistischen Operatoren benutzt):

$$
P_{\varphi} \rightarrow \text { const. } \sum_{m}\left|c_{l m}\right|^{2} P_{l m} \quad\left(P_{l m} \text { projiziert auf } \varphi_{l m}\right) .
$$

Dagegen führt in dem gewählten Falle die Messung der Observablen $A$, wenn man der Überlegung von LüDERs folgt, $\varphi$ in einen reinen Zustand über:

$$
P_{\varphi} \rightarrow \text { const. } P_{l} P_{\varphi} P_{l} \text {. }
$$

Entsprechend unterscheiden sich die beiden Ansätze, wenn kein reiner Zustand, sondern schon ein Gemisch in den Meßapparat einläuft, oder auch in dem Falle, wo zwar ein reiner Zustand einläuft, aber das Ergebnis nicht abgelesen wird.

So ergibt sich in dem letztgenannten Falle mit dem Ansatz von J. v. Neumann das Gemisch

$$
P_{\varphi} \rightarrow \sum_{l, m}\left|c_{l m}\right|^{2} P_{l m},
$$

mit dem Ansatz von LüDers jedoch

$$
P_{\varphi} \rightarrow \sum_{l} P_{l} P_{\varphi} P_{l}
$$

Im Falle, daß die Zustände als Systemgesamtheiten interpretiert werden, bedeuten diese Gemische, daß die statistischen Aussagen nicht über eine der Teilgesamtheiten, die man im Prinzip nach dem Meßergebnis aussondern könnte, gemacht werden, sondern über die ursprüngliche Gesamtheit nach der Messung. Der Erwartungswert für einen Operator $B$ wird mit dem Ansatz von J. v. NeumanN

$$
\sum_{l, m}\left|c_{l m}\right|^{2}\left(\varphi_{l m}, B \varphi_{l m}\right)
$$

mit dem Ansatz von LüDERS

$$
\sum_{l}\left(P_{l} \varphi, B P_{l} \varphi\right)
$$

Diese sind im allgemeinen verschieden, und es fragt sich, welcher Ansatz der richtige ist. Diese Frage kann wohl nur bei genauerer Kenntnis des jeweiligen Meßapparates beantwortet werden. Der Ansatz von Neumans unterstellt, daß die Phasenbeziehungen zwischen den ausgezeichneten Zuständen innerhalb eines zu einem Eigenwert $a_{7}$ gehörenden Unterraums gänzlich zerstört werden. Man kann fragen, ob dieser Umstand 
nicht erlaubte, den gleichen Meßapparat zur Messung einer Observablen, welche keine Entartung zeigt, heranzuziehen. Der Ansatz von LÜDERS dagegen behauptet, daß zwar eine Zerstörung der Phasenbeziehungen zwischen den Unterräumen $P_{k} \mathbf{H}$ stattfindet, daß aber die Phasenbeziehungen innerhalb jedes Unterraums völlig ungestört bleiben. Das bedeutet im allgemeinen wohl ebenfalls die Idealisierung eines Meßeingriffs.

Wie man an den Formeln erkennt, läßt sich die Gemischbildung nach LÜDERS im Falle eines selbstadjungierten Operators mit kontinuierlichem Spektrum gut verwenden, wenn man annimmt, daß der Apparat mit einer gewissen Unschärfe mißt. Dagegen würde in diesem Falle die Gemischbildung nach J. v. Neumans kompliziert und wohl erst durch den Übergang von einem Hilbertraum zu einem Gelfandschen Raumtripel [18] einer mathematischen Behandlung zugänglich.

Wir interessieren uns hier insbesondere für die Beziehungen zweier Observabler $A, B$, von denen wir annehmen, daß sie in zueinander raumartig gelegenen Gebieten $R_{1}$ bzw. $R_{2}$ gemessen werden und deshalb nach der Lokalitätsbedingung vertauschen sollen:

$$
[A, B]_{-}=0 .
$$

Für vertauschbare Observable kann man, falls man eine Gemischbildung nach LÜDERS durchführt, zwei Sätze beweisen. Wir wollen die Sätze formulieren und gleichzeitig die kurzen Beweise wiederholen, wobei hier auch auf Observable mit Streckenspektrum verallgemeinert wird.

Satz. a) Es seien $A$ und $B$ selbstadjungierte Operatoren, welche vertauschen: $[A, B]_{-}=0$. Dann gilt:

$$
\langle A\rangle_{\varphi}=\langle A\rangle_{B ; \varphi},\langle B\rangle_{\varphi}=\langle B\rangle_{A ; \varphi} .
$$

Dabei ist $\langle\cdots\rangle$ der Erwartungswert im Zustand $\varphi ; B ; \varphi$ bzw. $A ; \varphi$ sind die Gemische, die infolge der Messung von B bzw. A aus $\varphi$ ohne Ablesung entstehen. Betrachtet man Systemgesamtheiten $G^{\Sigma}$, so werden bei dieser Gemischbildung die Teilgesamtheiten nach der Messung wieder zusammengefaßt.

b) Gilt für jeden Zustand ${ }^{4}$

$$
\langle A\rangle_{\varphi}=\langle A\rangle_{B ; \varphi} \text { so folgt }[A, B]_{-}=0 .
$$

Dabei ist dahingehend idealisiert, daß, obwohl jeder einzelne Meßapparat nur ein endliches Auflösungsvermögen besitzt, alle Meßapparate zusammen eine beliebig feine Einteilung des Spektrums ermöglichen.

${ }^{4}$ Ergeben sich Schwierigkeiten mit Definitionsbereichen, gehe man zu beschränkten Funktionen über. 
Beweis für a). Es sei

$$
\begin{gathered}
\int_{-\infty}^{+\infty} d E_{B} \quad \text { die Zerlegung der Einheit für } B \\
\int_{-\infty}^{+\infty} d E_{B}=\sum_{j} \int_{J_{j}} d E_{B}=\sum_{j} P_{j} .
\end{gathered}
$$

Das Integral wird im Punktspektrum so zerlegt, daß jeweils ein Eigenzustand in jedem Integral erscheint, im kontinuierlichen Spektrum erfolgt die Zerlegung nach der Meßgenauigkeit des Apparates.

$$
\langle A\rangle_{\varphi}=(\varphi, A \varphi)=\left(\sum_{j} P_{j} \varphi, A \sum_{k} P_{k} \varphi\right)=\sum_{j}\left(P_{j} \varphi, A P_{j} \varphi\right) \text {. }
$$

Das letzte Gleichheitszeichen folgt wegen der Vertauschbarkeit der Spektralscharen. Es ist nun

$$
\sum_{j}\left(P_{j} \varphi, A P_{j} \varphi\right)=\langle A\rangle_{B ; \varphi} .
$$

Beweis für b). Es gilt

$$
\sum_{j}\left(\int_{J_{j}} d E_{B} \varphi, A \int_{J_{j}} d E_{B} \varphi\right)=\left(\sum \int_{j} d E_{B} \varphi, A \sum_{k} \int_{J_{k}} d E_{B} \varphi\right) .
$$

Wählt man $\varphi$ insbesondere so, daß es nur Komponenten in $J_{r}$ und $J_{s}$ besitzt, so folgt mit $\varphi=\varphi_{r}+\varphi_{s}$

$$
\left(\varphi_{r}, A \varphi_{s}\right)+\left(\varphi_{s}, A \varphi_{r}\right)=0 \text { oder }\left(\varphi_{r}, A \varphi_{s}\right) \text { rein imaginär. }
$$

Ersetzung von $\varphi_{r}$ durch $i \varphi_{r}$ zeigt, daß $\left(\varphi_{s}, A \varphi_{r}\right)=0$ sein muß. Wählt man eine Klasse von $\{\varphi\}$ so, daß $\varphi_{r}$ festbleibt, $\varphi_{s}$ alle Intervalle $J_{s}$ durchläuft, so folgt die Invarianz von $\int_{J_{r}} d E_{B}$ unter $A$. Da dieses Ergebnis für alle möglichen Einteilungen der Einheit in Intervalle gilt, folgt die Vertauschbarkeit von $A$ mit der Spektralschar von $B$, damit $[A, B]_{-}=0$.

Bemerkung. Die beiden Teile des Satzes gelten auch, wenn an Stelle von $\varphi$ ein Gemisch tritt.

Falls man eine Gemischbildung nach J. v. NeUmanN vornimmt, so findet man einfache Beispiele von selbstadjungierten Operatoren $A$ und $B$ mit $[A, B]_{-}=0$, so daß der Satz falsch wird.

Wie auch immer die Phasenbeziehungen zwischen den einzelnen Zuständen durch einen Meßeingriff erhalten bzw. zerstört werden mögen, aus dem folgenden wird klar, daß im Falle der Vertauschbarkeit von Observablen auf Grund der Lokalitätsbedingung nur die Gemische nach LÜDERS ein konsistentes Bild aufzubauen erlauben. Wir werden diese daher im folgenden bei Gemischbildungen verwenden.

Nunmehr soll mit Hilfe des Satzes a) eine Klassifizierung der Zustände vorgenommen werden. Wir gehen hier zu diesem Zweck von lokalen Observablen-Ringen aus, die man sich auch, sofern die Selbstadjungiert- 
heit der hermitischen Operatoren gewährleistet ist, durch Integration der Feldoperatoren und Polynombildung erzeugt denken kann. Jedem Element $O_{j}$ eines Systems von offenen Mengen $\left\{O_{k}\right\}$, welche den Minkowskiraum $E$ überdecken, ist eine Observablen-Algebra $\mathfrak{A}_{j}$ zugeordnet. Wir nehmen $(\mathscr{L})$ an, so daß mit $O_{r}$ raumartig zu $O_{s}$, für zwei beliebige Operatoren $A$ und $B$ mit $A \in \mathfrak{A}_{r}$ und $B \in \mathfrak{A}_{s}$ gilt $[A, B]_{-}=0$.

Man kann alle Zustände (unter ihnen auch alle Gemische), welche für eine lokale Observable $A$ und Funktionen dieser Observablen den gleichen Erwartungswert liefern, zu Klassen vereinigen (Zustände normiert). $\chi$ und $\psi$ seien Zustände, $\psi$ benutzen wir als Repräsentanten der Klasse.

Definition.

falls

$$
\chi \in K(A, \psi)
$$

$$
\langle h(A)\rangle_{\chi}=\langle h(A)\rangle_{\psi}
$$

gilt ( $h$ aus einem geeigneten Funktionenraum).

Im allgemeinen wird man jedoch nicht von einer Observablen ausgehen, sondern von einem lokalen Ring, so daß die Klassen kleiner werden. $\mathfrak{A}_{j}$ sei eine der oben eingeführten Observablenalgebren. Mit $\psi$ als Repräsentant definieren wir die Klasse zu $\mathfrak{A}_{j}$ und $\psi$ so:

Definition

falls

$$
\chi \in K\left(\mathfrak{U}_{j}, \psi\right)
$$

$$
\left\langle A_{l}\right\rangle_{\chi}=\left\langle A_{l}\right\rangle_{\psi} \text { für jeden Operator } A_{l} \in \mathfrak{A}_{j} .
$$

Bemerkung. Man sieht, daß z. B. jeder isometrische Operator $V$, der mit allen Operatoren aus $\mathfrak{U}_{j}$ vertauscht, die Klasse $K\left(\mathfrak{U}_{j}, \psi\right)$ invariant läßt. Man erkennt so, daß eine gewisse Verwandtschaft zu den von LICHT benutzten Klassen besteht [19]. Die Klasseneinteilung ist jedoch hier insbesondere hinsichtlich der Gemische getroffen, welche durch Messungen entstehen.

Lemma. $O_{r}$ und $O_{s}$ seien raumartig zueinander gelegen, $\mathfrak{A}_{r}$ sei der lokale Ring zu $O_{r}, A_{s}$ sei eine Observable aus $\mathfrak{A}_{s}$, das zu $O_{s}$ gehört. Es gilt für jedes $\varphi$

$$
A_{s} ; \varphi \in K\left(\mathfrak{U}_{r}, \varphi\right) \text {. }
$$

Wir erinnern daran, daß mit $A_{s} ; \varphi$ das Gemisch (nach LüDERs) bezeichnet ist, welches sich aus $\varphi$ infolge Messung von $A_{s}$ bildet. Wird $\varphi$ als Systemgesamtheit interpretiert, so ist $A_{s} ; \varphi$ als Zusammenfassung der Teilgesamtheiten nach der Messung zu lesen. Ist $\varphi$ ein Einzelsystem, so bedeutet $A_{s} ; \varphi$ die Zustandsbeschreibung nach der Messung bei unbekanntem Meßresultat.

Das Lemma ergibt sich aus der Lokalitätsbedingung und dem Satz von Abschnitt 8.

$2: 2$ Commun. math. Phys., Vol. 7 
Die Klassen $K(A, \psi)$ und auch noch die Klassen $K\left(\mathfrak{A}_{j}, \psi\right)$ sind, wie man auf Grund der vorangehenden Betrachtung sieht, sehr reich an Elementen. Sie sind jedoch für die Bemerkungen des nächsten $\mathrm{Ab}$ schnittes eng genug gefaßt. ${ }^{5}$

\section{Zustandsbeschreibung abhängig von der für den Beobachter möglichen Information}

Die Einsteinkausalität für klassische Systeme bedingt, daß Beobachter höchstens mit Lichtgeschwindigkeit von einem Meßergebnis Kenntnis erhalten können. Wir idealisieren dahingehend, daß die Beobachter tatsächlich mit Lichtgeschwindigkeit informiert werden und führen mit dieser Annahme, die mit $\left(\mathscr{C}_{k l}\right)$ abgekürzt wird, die Zustandsbeschreibung durch.

Das Verfahren soll zuerst an einem Beispiel erläutert werden: Bei $x^{*}$ und $y^{*}$ sollen Messungen der Observablen $A$ bzw. $B$ stattfinden. Die Messungen mögen die Werte $a_{l}$ bzw. $b_{k}$ ergeben; falls Streckenspektren vorliegen, sind die Werte durch kleine Intervalle zu ersetzen. Die Meßeingriffe sind zu punktförmigen Eingriffen (Raumzeit-Punkt) idealisiert. Der einlaufende Zustand sei $\psi$; alle Beobachter sind über das Meßprogramm unterrichtet.

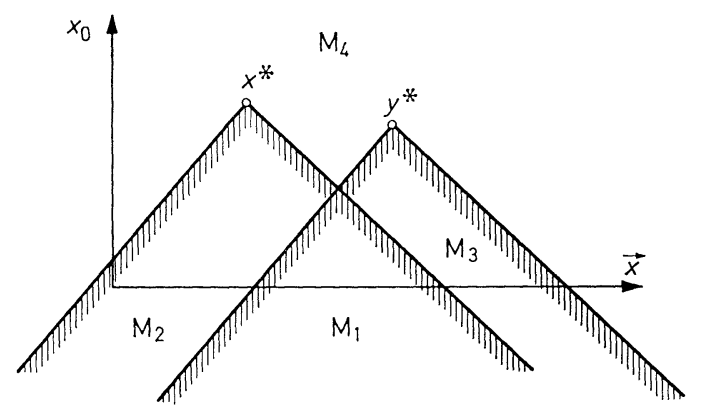

Fig. 4. $M$-Überdeckung im Falle zweier Messungen in zwei raumartig zueinander gelegenen Punkten

Die $M$-Bedeckung besteht aus 4 Mengen:

$$
\begin{array}{ll}
M_{1}=R_{x^{*}} \cap R_{y^{*}}, & M_{2}=R_{x^{*}} \cap\left(R_{y^{*}}\right)_{c} \\
M_{3}=\left(R_{x^{*}}\right)_{c} \cap R_{y^{*}}, & M_{4}=\left(R_{x^{*}}\right)_{c} \cap\left(R_{y^{*}}\right)_{c} .
\end{array}
$$

5 Den Vorschlag, in diesem Zusammenhang Gemische mit einzubeziehen, hat bereits auch Herr K. Kraus gemacht, wovon ich während der Drucklegung Kenntnis erhielt. Siehe K. Kraus: General Quantum Field Theories and Strict Locality, Zeitschrift für Physik 181, 1-12 (1964). 
Bemerkung. Falls $x^{*}$ zeitartig zu $y^{*}$ liegt, treten nur 3 Mengen auf; wir betrachten den interessanteren Fall, daß $x^{*}$ raumartig zu $y^{*}$ liegt.

Nunmehr fassen wir in $E$ liegende Gebiete gleicher Informationsmöglichkeit zusammen, welche wir eine $N$-Überdeckung nennen. Wenn wir die Vorwärtskegel mit $V$ bezeichnen (wir wollen sie ebenfalls als abgeschlossen betrachten), ergeben sich folgende Mengen:

$$
\begin{array}{ll}
N_{1}=\left(V_{x^{*}}\right)_{c} \cap\left(V_{y^{*}}\right)_{c}, & N_{2}=V_{x^{*}} \cap\left(V_{y^{*}}\right)_{c} \\
N_{3}=\left(V_{x^{*}}\right)_{c} \cap V_{y^{*}}, & N_{4}=V_{x^{*}} \cap V_{y^{*}} .
\end{array}
$$

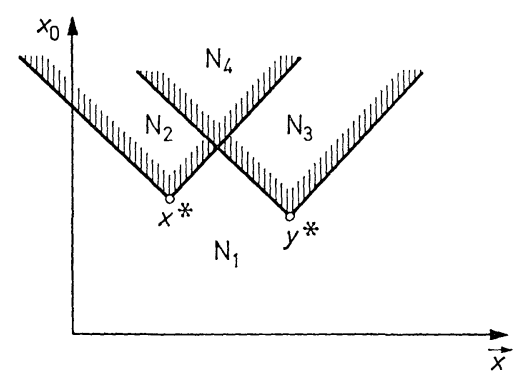

Fig. 5. $N$-Überdeckung im gleichen Beispiel

Eintragung der Meßergebnisse in die $N$-Überdeckung führt zu einer $N$-Karte; wir beziehen den bekannten Anfangszustand $\varphi$ mit ein:

$$
\left\{N_{1}: \varphi ; N_{2}: \varphi, P_{l}^{A} ; N_{3}: \varphi, P_{k}^{B} ; N_{4}: \varphi, P_{l}^{A}, P_{k}^{B}\right\} \text {. }
$$

Um Verwechslungen gegenüber einer $M$-Karte zu vermeiden, erscheinen die Informationen in den einzelnen Mengen $N_{j}$ hinter einem Doppelpunkt. Die Aussage ,Für $A$ wurde $a_{l}$ gemessen“, ist in $P_{l}^{A}$ zusammengefaßt, usw.

Nunmehr kann man die $M$-Karten angeben. Als Argument bei den Beobachtern $Z$ nach dem Übergang zu invarianten Grenzflächen der $M$-Gebiete - wie am Ende von Abschnitt 3 beschrieben - steht nur noch der Ort $u: Z(u)$. Beobachter, die ihren Ort in derselben Menge $N_{j}$ haben, erhalten für das System die gleiche $M$-Karte:

$Z(u)$ für $u \in N_{1}:\left\{P_{\varphi}\left(M_{1}\right), \sum_{j} P_{j}^{B} P_{\varphi} P_{j}^{B}\left(M_{2}\right), \sum_{j} P_{j}^{A} P_{\varphi} P_{j}^{A}\left(M_{3}\right)\right.$,

$$
\left.\sum_{j, m} P_{j}^{A} P_{m}^{B} P_{\varphi} P_{m}^{B} P_{j}^{A}\left(M_{4}\right)\right\}
$$

für $u \in N_{2}:\left\{P_{\varphi}\left(M_{1}\right), \sum_{j} P_{j}^{B} P_{\varphi} P_{j}^{B}\left(M_{2}\right)\right.$, const. $P_{l}^{A} P_{\varphi} P_{l}^{A}\left(M_{3}\right)$, const. $\left.\sum_{j} P_{l}^{A} P_{j}^{B} P_{\varphi} P_{j}^{B} P_{l}^{A}\left(M_{4}\right)\right\}$,

für $u \in N_{3}:\left\{P_{\varphi}\left(M_{1}\right)\right.$, const. $P_{k}^{B} P_{\varphi} P_{i}^{B}\left(M_{2}\right), \sum_{j} P_{j}^{A} P_{\varphi} P_{j}^{A}\left(M_{3}\right)$, const. $\left.\sum_{j} P_{j}^{A} P_{k}^{B} P_{\varphi} P_{k}^{B} P_{j}^{A}\left(M_{4}\right)\right\}$,

für $u \in N_{4}:\left\{P_{\varphi}\left(M_{1}\right)\right.$, const. $P_{k}^{B} P_{\varphi} P_{k}^{B}\left(M_{2}\right)$, const. $P_{l}^{A} P_{\varphi} P_{l}^{A}\left(M_{3}\right)$, const. $\left.P_{l}^{A} P_{k}^{B} P_{\varphi} P_{k}^{B} P_{l}^{A}\left(M_{4}\right)\right\}$. 
Die auftretenden Konstanten dienen der erneuten Normierung des Zustandes nach der Reduktion, welche bei Bekanntwerden des Meßergebnisses erfolgt. Auf Grund der Lokalitätsbedingung $(\mathscr{L})$ und da $x^{*}$ raumartig zu $y^{*}$ angenommen wurde, kann man die Projektoren von $A$ und $B$ miteinander vertauschen.

Die Verallgemeinerung der Methode auf kompliziertere Meßprogramme ist offensichtlich. Wir wollen die einzelnen Schnitte nur kurz skizzieren. Zur Ausgangssituation: Bei $x_{1}^{*}, x_{2}^{*}, \ldots x_{n}^{*}$ werden die Observablen $A_{1}, A_{2}, \ldots$ bzw. $A_{n}$ gemessen und bestimmte Werte als Meßresultate angenommen. Die Beobachter sind über einlaufenden Zustand und Meßprogramm unterrichtet. Zur Zustandsbeschreibung: Zuerst wird wie oben mittels Durchschnittsbildung aus den Rückwärtskegeln zu den Punkten $x_{1}^{*}, x_{2}^{*}, \ldots x_{n}^{*}$ bzw. aus deren Komplementärmengen die $M$-Überdeckung gewonnen. Die vom Ort des Beobachters abhängige $M$-Karte ergibt sich nach Konstruktion der $N$-Überdeckung und $N$-Karte. Die $N$-Überdeckung erhält man entsprechend der $M$-Überdeckung; an die Stelle der Rückwärtskegel treten die Vorwärtskegel zu den Punkten $x_{1}^{*}, x_{2}^{*}, \ldots x_{n}^{*}$. Beobachter $Z(u)$ mit Argumenten in derselben Menge $N_{j}$ besitzen die gleiche Systembeschreibung.

\section{Erwartungswerte für Observable}

Wie schon vorher sollen gelten

$(\mathscr{L})$ die Lokalitätsbedingung,

$(\mathscr{G})$ Gemischbildung nach LÜDERs,

$\left(\mathscr{C}_{k l}\right)$ Einstein-Kausalität für die Übermittlung von Meßergebnissen.

Zunächst sei wie im vorigen Abschnitt das Meßprogramm fixiert: Bei $x_{1}^{*}, x_{2}^{*}, \ldots x_{n}^{*}$ werden die Observablen $A_{1}, A_{2}, \ldots$ bzw $A_{n}$ gemessen; $P_{l_{1}}^{1}, P_{l_{2}}^{2}, \ldots$ bzw. $P_{l_{n}}^{n}$ sind die zugehörigen Projektoren, welche die angenommenen Resultate beschreiben. $P_{l_{j}^{j}}^{j}$ projiziert auf den Unterraum zum Eigenwert $l_{j}$ von $A_{j}$ oder auf einen Unterraum zum Intervall $I_{j}$ der Spektralzerlegung des Einheitsoperators für $A_{j}$, falls ein Streckenspektrum vorliegt. Der Anfangszustand sei $\varphi$.

In welcher Weise berechnet ein bei $u$ befindlicher Beobachter $Z$ den Erwartungswert für eine der Observablen, z. B. für $A_{k}$ ? Interessiert er sich für den Erwartungswert einer Einzelmessung an einem System $\Sigma$, so kann man annehmen, daß $u \in\left(V_{x_{k}^{*}}\right)_{c}$ ist. Will man dagegen den Mittelwert für $A_{k}$ über eine Systemgesamtheit $G^{\Sigma}\left(u, \ldots, l_{s}, \ldots\right)$ berechnen, welche durch den gleichen Anfangszustand $\varphi$ und gleiche Meßresultate $l_{s}$ für die Operatoren $A_{s}$ mit $x_{s}^{*} \in R_{u}$ gekennzeichnet ist, so kann die Frage auch für $u \in V_{x_{k}^{*}}$ sinnvoll sein. $Z(u)$ benutzt zur Berechnung des Erwartungswertes von $A_{k}$ die relevante, d. h. von $u$ abhängige $M$-Karte. Dieser Karte entnimmt er den Zustand wie er vor der Messung von $A_{k}$ 
vorlag, und bildet mit ihm den Erwartungswert, d. h. er benutzt den Zustand, der zu der Menge $M_{j}$ gehört, welche den $x_{k}^{*}$ enthaltenden Teil von $R_{x_{k}^{*}}$ überdeckt. Den auf diese Weise gebildeten Erwartungswert bezeichnen wir mit dem folgenden Symbol:

$$
\left\langle A_{k}\right\rangle_{u ;[\ldots] ; \varphi}
$$

$[\cdots]$ ist eine Abkürzung für das Meßprogramm und für die angenommenen Meßresultate; von diesen wird wie oben angegeben in Abhängigkeit von $u$ (natürlich wird auch nicht das Resultat der Messung von $A_{k}$ benutzt) nur ein Teil verwendet. Diese Tatsache ist ein Ausdruckdafür, daß auf zwei verschiedenen Stufen Aussagen erfolgen: Erstens die Beschreibung des Systems durch einen Beobachter $Z(u)$, zweitens Aussagen über Relationen der Beschreibungen für verschiedene Beobachter, welche der eigentliche Gegenstand dieser Bemerkungen sind.

Man kann den folgenden Satz beweisen:

Satz. Es gilt

$$
\left\langle A_{k}\right\rangle_{u ;[\ldots] ; \varphi}=\left\langle A_{k}\right\rangle_{u ;[\ldots]^{\prime} ; \varphi} .
$$

Dabei erscheinen in $[\cdots]^{\prime}$ nur noch die Operatoren $A_{j_{1}}, \ldots, A_{j_{r}}$ und a'eren Meßresultate $l_{j_{1}}, \ldots l_{j_{r}}$ aus $[\cdots]$, deren zugehörige $x_{j s}^{*}($ mit $s=1,2, \ldots r)$ die Bedingung erfüllen:

$$
x_{i s}^{*} \in R_{x_{i}^{*}}^{*} \cup R_{u} .
$$

Zusatz. Insbesondere gilt für jedes $u \in N_{1}$ ( $N_{1}$ ist die $N$-Menge, in der die zugehörigen Beobachter noch keine Meßresultate besitzen) und für alle Observablen $A_{l}$ mit $x_{l}^{*} \in\left(V_{x_{j}^{*}}\right)_{c}$ für alle $j \neq l$ :

$$
\left\langle A_{l}\right\rangle_{u ;[\ldots] ; \varphi}=\left\langle A_{l}\right\rangle_{\varphi} .
$$

Der Satz sagt also aus, daß eine Beeinflussung des Erwartungswertes einer Observablen $A_{k}$ durch Messung von anderen Observablen nur dann stattfinden kann, wenn deren Messung im Rückwärtskegel zu $x_{k}^{*}$ stattfindet, oder wenn das Meßresultat der anderen Observablen dem Beobachter $Z(u)$ bekannt ist.

Es ist klar, daß der Zusatz ein Spezialfall des Satzes ist, da wegen $u \in N_{1}$ dem Beobachter $Z(u)$ keine Meßresultate bekannt sind und wegen $x_{l}^{*} \in\left(V_{x_{j}^{*}}\right)_{c}$ für alle $j \neq l$ keine Messungen im Rückwärtskegel zu $x_{l}^{*}$ stattfinden.

Beweis des Satzes. Gibt es kein $x_{r}^{*} \in\left(R_{x_{k}^{*}} \cup R_{u}\right)_{c}$, so ist nichts zu beweisen.

Liege nunmehr

$$
x_{r}^{*} \in\left(R_{x_{i}^{*}}^{*} \cup R_{u}\right)_{c}=\left(R_{x_{k}^{*}}\right)_{c} \cap\left(R_{u}\right)_{c} .
$$

Man zeigt zuerst, daß

$$
\left\langle A_{k}\right\rangle_{u ;\left[\ldots ; l_{r-1}, A_{r-1} ; l_{r}, A_{i} ; l_{r+1}, A_{r+1} ; \ldots\right] ; \varphi}=\left\langle A_{k}\right\rangle_{u ;\left\{\ldots, l_{r-1}, A_{r-1} ; l_{r+1} . A_{r+1} \ldots\right] ; \varphi}
$$


gilt. Wir können annehmen, daß $x_{r}^{*} \in\left(V_{x_{k}^{*}}\right)_{c}$ liegt. Andernfalls gehen $l_{r}$ und $A_{r}$ in den Erwartungswert gar nicht ein; siehe dazu die Konstruktion der $M$-Karte im vorangehenden Abschnitt. $x_{r}^{*}$ liege nunmehr raum$\operatorname{artig}$ zu $x_{k}^{*}$. Von den übrigen $x_{s}^{*}, s \neq k$, können wir ohne Verlust der Allgemeinheit auch annehmen, daß $x_{s}^{*} \in\left(V_{x_{*}^{*}}\right)_{c}$ gilt; andernfalls findet durch die Messung der zugehörigen Observablen $A_{s}$ keine Zustandsänderung im Punkte $x_{k}^{*}$ statt.

Wir benutzen die folgenden Bezeichnungen: Wie vorher ist $A ; \varphi$ das Gemisch, das durch Messung von $A$ aus $\varphi$ entsteht, ohne Ablesung des Meßergebnisses (bzw. wenn das Meßergebnis wegen $\left(\mathscr{C}_{k l}\right)$ nicht bekannt ist). $B ; A ; \varphi$ ist das Gemisch, das durch Messung von $B$ aus $A$; $\varphi$ entsteht, ebenfalls ohne Ablesung des Meßergebnisses für $B$; entsprechend bei mehr als 2 Observablen. Bei Ablesung des Meßergebnisses für $A$ schreiben wir (bzw. der Meßergebnisse für $A$ und $B$ )

$$
l_{A}, A ; \varphi \text { bzw. } l_{B}, B ; l_{A}, A ; \varphi,
$$

wenn $A$ und $B$ gemessen wurden; entsprechend bei mehr als zwei Observablen. Wollen wir keine Entscheidung treffen, ob eine Reduktion der Gemische vorgenommen werden soll oder nicht, so setzen wir die Meßresultate in Klammern, z. B.

$$
\left(l_{B}\right), B ;\left(l_{A}\right), A ; \varphi \text {. }
$$

Letztere Schreibweise eignet sich, um die belegenden Zustände einer $M$-Überdeckung zu bezeichnen; in Abhängigkeit von $u$ für $Z(u)$ sind dann gewisse Meßresultate zu setzen oder wegzulassen. Es gilt also

$$
\begin{aligned}
u ; & {\left[\ldots ; l_{r-1}, A_{r-1} ; l_{r}, A_{r} ; l_{r+1}, A_{r+1} ; \ldots\right] ; \varphi } \\
& =\ldots\left(l_{r-1}\right), A_{r-1} ;\left(l_{r}\right), A_{r} ;\left(l_{r+1}\right), A_{r+1} ; \ldots ; \varphi .
\end{aligned}
$$

Wegen der Annahme $x_{s}^{*}$ mit $s \neq k$ raumartig zu $x_{k}^{*}$ treten hier rechts tatsächlich alle Observable außer $A_{k}$ auf.

Da $x_{r}^{*} \in\left(R_{x_{k}^{*}}\right)_{c} \cap\left(R_{u}\right)_{c}$ mithin $x_{r}^{*} \in\left(R_{u}\right)_{c}$ ist, gilt

$$
\begin{aligned}
\ldots ;\left(l_{r-1}\right), A_{r-1} ;\left(l_{r}\right), A_{r} ;\left(l_{r+1}\right), A_{r+1} ; \ldots ; \varphi & \\
& \rightarrow \ldots ;\left(l_{r-1}\right), A_{r-1} ; A_{r} ;\left(l_{r+1}\right), A_{r+1} ; \ldots ; \varphi,
\end{aligned}
$$

d. h. das Ergebnis der Messung von $A_{r}$ ist $Z(u)$ nicht bekannt. Wegen $(\mathscr{L})$ kann man $A_{r}$ über die Operatoren, welche $Z(u)$ bekannte Meßresultate liefern, nach links schieben; dann folgt nach Satz a) aus Abschnitt 4:

$$
\begin{aligned}
& \ldots ;\left(l_{r-1}\right), A_{r-1} ; A_{r} ;\left(l_{r+1}\right), A_{r+1} ; \ldots ; \varphi \in \\
& \quad \in K\left(A_{l}, \ldots ;\left(l_{r-1}\right), A_{r-1} ;\left(l_{r+1}\right), A_{r+1} ; \ldots ; \varphi\right) .
\end{aligned}
$$

Damit wird

$$
\left.\left\langle A_{k}\right\rangle_{u} ; \ldots ; l_{r-1}, A_{r-1} ; l_{r}, A_{r} ; l_{r+1}, A_{r+1} ; \ldots\right] ; \varphi=\left\langle A_{k}\right\rangle_{u} ;\left[\ldots ; l_{r-1}, A_{r-1} ; l_{r+1}, A_{r+1} ; \ldots\right] ; \varphi .
$$


Wenn es keinen weiteren Punkt $x_{s}^{*}, s \neq r, s \neq k$ mit $x_{s}^{*} \in\left(R_{x_{i}^{*}} \cup R_{u}\right)_{c}$ gibt, ist der Beweis erbracht. Andernfalls kann man mit

$$
\left\langle A_{k}\right\rangle_{u ;\left[\ldots ; l_{r-1}, A_{r-1} ; l_{r+1}, A_{r+1} ; \ldots\right] ; \varphi}
$$

beginnend weitere Observable aus [...] eliminieren und so schließlich zu $\left.\left\langle A_{k_{i}}\right\rangle_{u ;[\ldots]}\right]^{\prime} ; \varphi$ gelangen.

\section{Erwartungswerte bei nicht-fixiertem Meßprogramm}

Dic bisherige Diskussion wurde in der Weise geführt, daß nicht nur die Elemente des Hilbertraumes als Zustände eines Systems in ihrer ganzen Erstreckung im vierdimensionalen Raume gedeutet wurden, sondern daß auch das Meßprogramm als ein Objekt ,sub specie aeternitatis" erschien. Es sollte jedoch noch eine andere Auffassung hinsichtlich der auszuführenden Messungen am System möglich sein. Die Berechnung des Erwartungswertes einer Größe sollte erlaubt sein, auch wenn nicht gewiß ist, ob die Messung tatsächlich erfolgt oder nicht. Berechnung des Erwartungswertes einer Größe und die Verifizierung durch die Messung (oder eine Meßreihe) sind durchaus getrennte Akte.

Wenn man sich nicht bei solchen nur als möglich gedachten Messungen in Widersprüche verwickeln will, müssen gewisse einfache Bedingungen eingehalten werden.

1. Welche Messungen als möglich und nicht als fixiert behandelt werden können, hängt vom Orte des Beobachters ab. Es sei $Z(u)$ der Beobachter am Orte $u$, der nunmehr ebenfalls als aktiver Experimentator $Z_{e}$ wirken kann. Für ihn kommt als Raumteil für mögliche Experimente nur $V_{u}$ in Frage: $Z_{e}\left(x^{*}\right)$ mit $x^{*} \in V_{u}$.

2. Weiter sei $w \in\left(R_{x^{*}}\right)_{c}$. Dann ergibt sich für $Z\left(w, \mathbf{v}_{r}\right), w$ fest, $\mathbf{v}_{r}$ variabel nur in 2 Fällen ein konsistentes Bild:

a) Die Messung bei $x^{*}$ ist geschehen oder wird mit Notwendigkeit in Zukunft geschehen (Perfekt oder Futur hängt von $\mathbf{v}_{r} a b$ ).

b) Die Messung bei $x^{*}$ ist unterblieben oder wird mit Notwendigkeit unterbleiben.

Man hat sich also, um mit der Fragestellung in Übereinstimmung zu bleiben, auf $Z(w)$ mit $w \in R_{x^{*}}$ zu beschränken.

Erfüllt, umgekehrt, ein Beobachter $Z(w)$ die Bedingung $w \in R_{x^{*}}$, so kommt wegen $x^{*} \in V_{w} Z(w)$ als aktiver Experimentator im Punkte $x^{*}$ in Frage, $Z(w) \rightarrow Z_{e}\left(x^{*}\right)$.

Aus dieser Betrachtung resultiert das Folgende: Wenn man für eine $M$-Karte, die zum Anfangszustand $\phi$ und den Messungen der Observablen $A_{1}, A_{2}, \ldots$ bzw. $A_{n}$ an den Punkten $x_{1}^{*}, x_{2}^{*}, \ldots$ bzw. $x_{n}^{*}$ gehört, die Messung von $A_{r}$ am Punkte $x_{r}^{*}$ nicht als faktisch, sondern nur als möglich behandeln will, so sind nur $Z(u)$ mit $u \in R_{x_{r}^{*}}$ zugelassen. $R_{x_{r}^{*}}$ ist andererseits nach Abschnitt 3 genau das Gebiet, in dem die belegenden 
Zustände, durch die Messung von $A_{r}$, unbeeinflußt sind. Als Meßwerte, die $Z(u)$ mit $u \in R_{x_{r}^{*}}$ zur Verfügung stehen, kommen nur die in Frage, welche zur Messung von $A_{j}, \ldots A_{j l}$ gehören, mit $x_{j k}^{*} \in R_{u}$. Wegen $R_{u} \subset R_{x_{r}^{*}}$ ändern sich diese Meßwerte nicht, gleichgültig, ob $A_{r}$ gemessen wird oder nicht. Dieses Resultat ist in Übereinstimmung mit der Ausgangslage, daß $Z(u)$ die Messung bei $x_{r}^{*}$ als eine mögliche interpretiert.

Entsprechend kann man mehrere Messungen des Programms als mögliche Messungen interpretieren, wobei sich im allgemeinen eine weitere Einschränkung des Gebietes der zugelassenen Beobachter ergibt. Beachtet man diesen Umstand, so kann man eine $N$-Karte und die nunmehr eingeschränkte Mannigfaltigkeit der zugehörigen $M$-Karten auch für die abgeänderte Fragestellung benutzen.

Zum Schluß dieses Abschnittes soll noch eine weitere Variante eines nicht-fixierten Meßprogramms diskutiert werden. Wir nehmen an, $Z(u)$ befinde sich am Punkte $x_{l}^{*}$, an dem $A_{l}$ gemessen wird $Z(u)=Z_{e}\left(x_{l}^{*}\right)$. Wir wollen der Einfachheit halber fordern, daß sich in $V_{x_{l}^{*}}$ keiner der Punkte $x_{s}^{*} r=1, \ldots, l-1, l+1, \ldots n$ befindet. Wie man sich überlegt, ändert diese Vereinfachung am abzuleitenden Resultat nichts. Nunmehr werde den Experimentatoren $Z_{e}\left(x_{s}^{*}\right)$ bei allen $x_{s}^{*}$ gestattet (wobci $x_{s}^{*} \in\left\{x_{r}^{*}\right\}$ und $x_{s}^{*}$ raumartig zu $x_{l}^{*}$ ) an Stelle der Größen $A_{s}$ beliebige andere Größen $C_{s}$ zu messen oder auch eine Messung bei $x_{s}^{*}$ ganz zu unterlassen.

Wir stellen die Frage, ob $Z_{e}\left(x_{l}^{*}\right)$ durch die Messung von $A_{l}$ einen Hinweis dafür erhält, zu welcher Wahl sich die $Z_{e}\left(x_{s}^{*}\right)$ entschlossen haben.

Nach dem Satz aus Abschnitt 6 ist die Frage zu verneinen; man sicht das sofort, wenn man sinngemäß

$$
R_{x_{k}^{*}}=R_{x_{l}^{*}} \quad \text { und } \quad R_{u}=R_{x_{l}^{*}} \text { setzt. }
$$

Die in Abschnitt 3 aufgezählten Beispiele können als einfaches Anschauungsmaterial für die angegebenen Grundsituationen bei fixiertem und nichtfixiertem Meßprogramm dienen.

\section{Zur Lokalitätsbedingung und einige weitere Bemerkungen}

Den Antrieb zu diesen Überlegungen gab die Frage, ob die Lokalitätsforderung für relativistische Quantenfelder einleuchtend ist oder nicht. Wir haben versucht, die Frage möglichst weit durch die Betrachtung von Meßeingriffen am System zu verfolgen. Die Betrachtungen werden dort irrelevant, wo ein Feld nicht mehr selbst als observabel, sondern nur als Hilfsgröße für die Berechnung von Observablen angesehen wird.

Sieht man das Feld als observabel an, so wird die Bedeutung der Lokalitätsforderung sichtbar : 
1. Wenn man, wie im Abschnitt 5 geschehen, eine Zustandsbeschreibung für ein System, an dem Messungen vorgenommen werden, geben will, so gerät man ohne Lokalitätsbedingung sofort in Widersprüche. Wir wollen das z. B. im Falle zweier Messungen von $A$ bzw. $B$ bei $x^{*}$ bzw. $y^{*}$ mit $x^{*}$ raumartig zu $y^{*}$ zeigen. Hierzu braucht man sich nur an das bisher in den Hintergrund getretene Relativitätsprinzip für die $\mathrm{Zu}$ stände zu erinnern. Bisher beschrieben wir die Zustände und ihre Veränderungen für Beobachter an verschiedenen Raumpunkten und in verschiedenen Bewegungszuständen stets im gleichen festen $x$-Koordinatensystem.

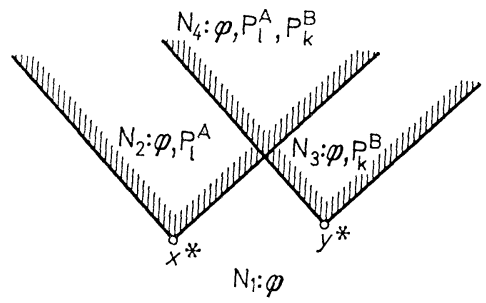

Fig. 6. $N$-Karte im $x$-System

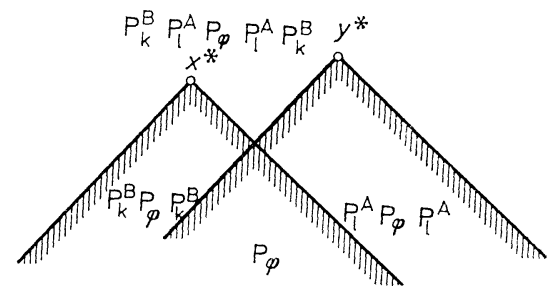

$M$-Karte im $x$-System für $u \in N_{4}$

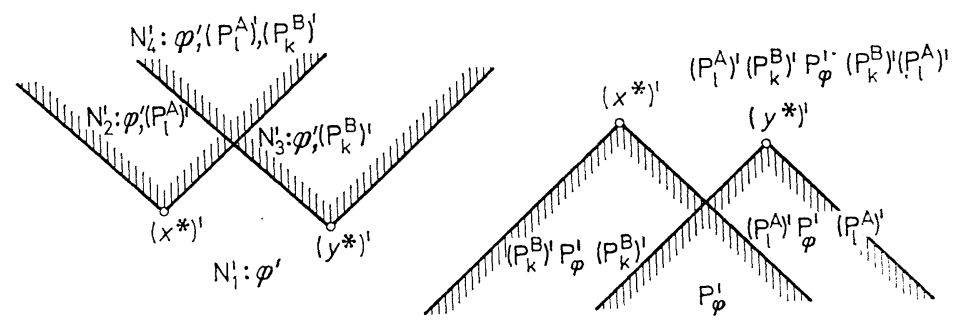

Fig. 7. $N$-Karte und $M$-Karte im $x^{\prime}$-System

Geht man zu einem $x^{\prime}$-System über, so erhält man mit geeignetem $A, a$ und $\varphi^{\prime}=u(\Lambda, a) \varphi, A^{\prime}=u(\Lambda, a) A u^{-1}(\Lambda, a), B^{\prime}=\ldots$ ( $(\Lambda$ homogene Lorentz-Transformation, $a$ Verschiebung) die $N$-Karte im $x^{\prime}$-System und $M$-Karte im $x^{\prime}$-System für $u \in N_{4}^{\prime}$.

Wenn die Gebiete der $M$-Karte und ihre Belegungen einen vom Koordinatensystem ( $x$ - oder $x^{\prime}$-System) unabhängigen Sinn haben sollen, so muß zu $M_{4}^{\prime}$ die transformierte Belegung von $M_{4}$ gehören:

$$
\left(P_{k}^{B}\right)^{\prime}\left(P_{l}^{A}\right)^{\prime} P_{\varphi}^{\prime}\left(P_{l}^{A}\right)^{\prime}\left(P_{k}^{B}\right)^{\prime} .
$$

Andererseits gehört zu $M_{4}^{\prime}$ die Belegung:

$$
\left(P_{l}^{A}\right)^{\prime}\left(P_{k}^{B}\right)^{\prime} P_{\varphi}^{\prime}\left(P_{k}^{B}\right)^{\prime}\left(P_{l}^{A}\right)^{\prime},
$$


wenn man von der transformierten $N$-Karte ausgeht. Hieraus folgt, da die Gleichheit für beliebige $l$ und $k$ bestehen muß

$$
[A, B]_{-}=0 \text {. }
$$

Das Bestehen der Lokalitätseigenschaft macht erst eine mit dem Relativitätsprinzip (für die Zustände) verträgliche Beschreibung der Zustandsänderungen durch Messung möglich.

2. Wir nehmen die Einstein-Kausalität für klassische Systeme $\left(\mathscr{C}_{k l}\right)$ und die gewählte Gemischbildung $(\mathscr{G})$ als relevant an. Welche weiteren Konsequenzen folgen dann aus der Lokalität? Aus der Diskussion am Ende des vorigen Abschnitts geht hervor, daß die Erwartungswerte für eine Observable für einen Beobachter am Orte der Messung $x^{*}$ dieser Observablen nicht davon abhängen, welche anderen Observablen oder ob überhaupt andere Observable an raumartig zu $x^{*}$ gelegenen Punkten gemessen werden. So vermag im Beispiel von Boнm und AHARonov ein Experimentator, der am Teilchen $C$ eine Spinrichtung mißt, die ihm freie Wahl der Richtung des Magnetfeldes nicht dazu auszunutzen, eine Nachricht an den raumartig entfernten Beobachter am Teilchen $D$ zu übermitteln. Dieses wäre im Prinzip möglich, wenn die Erwartungswerte einer Messung an $D$ für einen Beobachter am Ort von $D$ von der Messung an $C$ abhingen.

Wir abstrahieren von diesen Bemerkungen: Teilt man die Beobachter (bzw. Meßapparate) vom beobachteten System ab, so sind für die Übermittlung des Meßergebnisses von einem Experimentator $Z_{e}\left(u_{1}\right)$ zu einem zweiten Experimentator $Z_{c}\left(u_{2}\right)$ zwei grundsätzlich verschiedene Wege I und II denkbar. Der Weg I verläuft auf der mit den Begriffen der klassischen Physik zu beschreibenden Seite der Beobachter und Meßgeräte. Der Weg II bedient sich der Veränderung des Systems infolge der Messung. Eine solche Veränderung weist scheinbar akausale Züge auf. Trotzdem kann II nicht zur Brechung der Einstein-Kausalität $(\mathscr{C})$ verwendet werden, solange für alle Wege vom Typ I die EinsteinKausalität $\left(\mathscr{C}_{k l}\right)$ gilt, und solange man an der Lokalitätseigenschaft festhält.

Nach dem zweiten Teil des Satzes in Abschnitt 8 gilt auch die Umkehrung: Soll keine Beeinflussung des Erwartungswertes einer Observablen in allen möglichen Zuständen des Systems durch die Messung: einer anderen Observablen möglich sein, so müssen die Observablen vertauschen (Gemischbildung nach LüDERS vorausgesetzt). Soll insbesondere keine Beeinflussung des Erwartungswertes einer Observablen durch Messung von Observablen, welche raumartig zur betrachteten Observablen liegen, stattfinden, so hat man $(\mathscr{L}) \mathrm{zu}$ fordern. Es ergeben sich damit die folgenden Implikationen:
$\left(\mathscr{C}_{k l}\right)$ und $(\mathscr{G})$ und
$(\mathscr{L}) \Rightarrow(\mathscr{C})$
$(\mathscr{C})$ und $(\mathscr{G})$
$\Rightarrow(\mathscr{L})$. 
Wir möchten uns aber hüten, dieses Ergebnis so zu deuten, als sei unter den Annahmen $(\mathscr{C})$ und $(\mathscr{G})$ die Eigenschaft $(\mathscr{L})$ tatsächlich bewiesen. Die angestellten Überlegungen und Beweise arbeiten mit scharfen Gebietsgrenzen der Mengen $N_{j}$ bzw. $M_{j}$, was punktförmige Meßeingriffe voraussetzt. Tatsächlich finden Meßeingriffe in vierdimensionalen Gebieten des Minkowskiraumes statt, welche infolge der Größe der Meßapparate nicht beliebig klein sein können. Die Überlegungen sind damit nur soweit relevant, wie der Abstand der Punkte nicht unter das Auflösungsvermögen der Meßapparate sinkt. Immerhin ist eines ersichtlich: Falls man die Lokalitätsbedingung $(\mathscr{L})$ aufgibt, so muß man eine andere Bedingung $\left(\mathscr{L}_{e}\right)$ mit schwer definierbaren Eigenschaften stellen, wenn man eine noch als konventionell anzusehende Systembeschreibung erhalten will. Betrachtet man weiter $(\mathscr{C})$ als richtig, so müßte auch $\left(\mathscr{L}_{e}\right)$ im Falle auflösbarer Abstände im vierdimensionalen Raum gestatten, aus $\left(\mathscr{C}_{k \imath}\right)$ die Einstein-Kausalität $(\mathscr{C})$ abzuleiten.

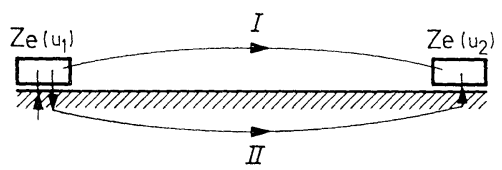

Fig. 8. Die Wege I und II für die Übermittlung des Meßergebnisses

Wir haben uns nicht mit der Erzeugung des Anfangszustandes auseinandergesetzt. Gewisse Fragen, die sich hierbei stellen, findet man z. B. bei Liснт [20] beantwortet. Wir wollen die Betrachtung mit einer kurzen Bemerkung zu diesem Problem beenden. ${ }^{6}$

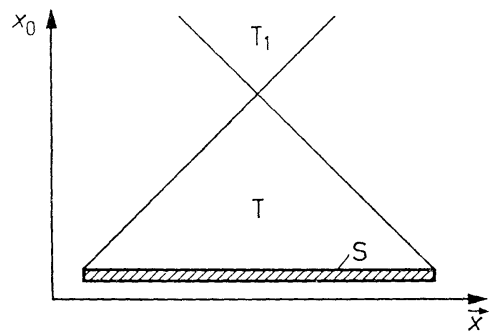

Fig. 9. Zur Präparierung eines Zustandes

${ }^{6}$ Herr K. Kraus, Univ. Marburg, hat mir während der Drucklegung dieser Arbeit freundlicherweise Teile seiner Habilitationsschrift zugänglich gemacht. Darin werden einige Fragen, die mit dem Gegenstand dieser Arbeit zusammenhängen, eingehender diskutiert. Insbesondere findet man auch zum Problem der Präparierung von Zuständen eine Reihe von Bemerkungen. 
Gegeben sei ein Stück $S$ einer Haagschen Schicht; zu $S$ gehört ein gewisser Observablenring. Man kann versuchen, durch Messungen, welche $\mathrm{zu}$ einer maximal-Abelschen Algebra dieses Observablenringes gehören, einen Zustand zu präparieren, der im Gebiet $T$ einem reinen Zustand nahekommt. Der so präparierte angenähert reine Zustand ist jedoch nur Beobachtern in $T_{1}$ bekannt; $T_{1}$ wiederum ist unkontrollierten Einflüssen, die von Gebieten außerhalb $S$ stammen, ausgesetzt.

Den Herren R. HaAg und A. S. Wightman bin ich für Diskussionen, welche dem hier gemachten Lösungsvorschlag vorausgingen, zu großem Dank verpflichtet. Mein Dank gilt auch den Herren Dürr, ReeH, Rinke, Roos und SwIEct für fördernd wirkendes Interesse.

\section{Literatur}

1. Siehe z. B. Wightuan, A. S.: Problèmes mathématiques de la théorie quantique des champs. Vorlesungsausarbeitung. Paris 1958.

2. Siehe z. B. Wightman, A. S.: Introduction to some aspects of the relativistic dynamics of quantized fields. Lectures at the French Summer School of Theoretical Physics, Cargèse, Corsica 1964.

3. Siehe z. B. HAAG, R., and D. Kastler: An algebraic approach to quantum field theory. J. Math. Phys. 5, 848-861 (1964).

Arakr, H.: On the algebra of all local observables. Progr. Theoret. Phys. (Kyoto) 32, $844-854$ (1964).

Guenin, M., and B. Misra: On the von Neumann algebras generated by field operators. Nuovo Cimento 30, 1272-1290 (1963).

4. HaAG, R., and D. KastLer: In [3].

5. ReeH, H., u. S. Schlieder: Bemerkungen zur Unitäräquivalenz von Lorentzinvarianten Feldern. Nuovo Cimento 22, 1051-1068 (1961).

Borchers, H. J.: On the structure of the algebra of field operators. Nuovo Cimento 24, 214-236 (1962).

6. - , and W. Zimmermana: On the self-adjointness of field operators. Nuovo Cimento 31, 1047-1059 (1964).

7. Siehe z. B. Feynan, R. P.: Space-time approach to quantum electrodynamics. Phys. Rev. 76, 769-789 (1949).

WightMAN, A. S.: In [2].

Jost, R., u. K. Hepp: Matrixelemente des Translationsoperators. Helr. Phys. Acta 35, 34-46 (1962).

8. LüDers, G.: Über die Zustandsänderung durch den Meßprozeß. Ann. Physik 6. Folge Bd. 8, 322-328 (195̃1).

9. Siehe z. B. Süssmann, G.: Über den Meßvorgang. Abhandl. Bayer. Akad. Wiss. Math.-Naturw. Kl. 1958.

Wigner, E. P.: The problem of measurement. Am. J. Phys, 31, 6-15 (1963).

JAUCH, J. M.: The problem of measurement in quantum mechanics. Helv. Phys. Acta 37, 293-316 (1964).

10. Scherbe, E.: Die kontingenten Aussagen in der Physik. Frankfurt am Main und Bonn: Athenäum-Verlag 1964.

11. Einstein, A., B. Podolsky, and N. Rosex: Can quantum-mechanical description of physical reality be considered complete? Phys. Rev. 47, 777-780 (1935). 
12. z. B. BeLL, J. S.: On the Einstein-Podolsky-Rosen paradox. Physics 1, 195 to 200 (1964).

13. Bohm, D., and Y. Aharonov: Discussion of experimental proof for the paradox of Einstein, Rosen, and Podolsky. Phys. Rev. 108, 1070-1076 (1957).

14. z. B. Wightman, A. S.: Note on polarization effects on Compton scattering. Phys. Rev. 74, 1813-1817 (1948).

15. ๘. B. Nishisma, K.: Fundamental particles. New York and Amsterdam: W. A. Benjamin Inc. 1963.

16. DAy, T. B.: Demonstration of quantum mechanics in the large. Phys. Rev. 121, 1204-1206 (1961).

17. z. B. Neumand, J. v.: Mathematische Grundlagen der Quantenmechanik. Kap. VI. 1. Berlin: Julius Springer 1932.

18. Gelfand, I. M., u. N. J. Wilenkix: Verallgemeinerte Funktionen, Bd. IV. Berlin: Deutscher Verlag der Wissenschaften 1964.

19. Licht, A. L.: Superposition of local states. Bull. Am. Phys. Soc. 10, no 1, 47 (1965); siehe auch 20.

20. - Local states. J. Math. Phys. 7, 1656-1669 (1966).

Dr. S. Schlieder

Max-Planck-Institut

f. Physik und Astrophysik

8000 München, Föhringer Ring 6 